\section{POSTER 061.}

\section{Epidemiology of Pediatric EMS Practice: A Multi-State Analysis}

Steven M. Joyce, $M D$, * Douglas Brown, CEP, Elizabeth Nelson

Division of Emergency Medicine, University of Utah,

Salt Lake City Utah, Utah USA

Purpose: Describe the epidemiology of pediatric EMS practice in a large patient population over a wide geographic range.

Methods: Retrospective computer analysis of EMS databases from four states using a common system. All records of patients $\leq 14$ years old entered during 1990-1992 were analyzed for demographics, elapsed prehospital times, incident descriptors, illness/injury and treatment delivered.

Results: A total of $1,512,907$ records was reviewed. A total of 61,132 pediatric cases comprised $4 \%$ of the prehospital population. Fifty-six percent were male, and children 7-14 comprised $46 \%$ of the cases. Children were transported by ambulance in $89 \%$ of runs, and care was refused in $8 \%$. Mean response time was $9 \pm 16$ minutes; scene time $12 \pm 14$ minutes; and transport time $14 \pm 20$ minutes with median elapsed times of 6,10 , and 9 minutes respectively. Sixty percent of incidents were traumatic, with motor-vehicle accidents and falls most common. Blunt injuries accounted for $94 \%$ of trauma, while respiratory distress, seizures, and poisoning/overdose were the most common medical problems. Vital signs were obtained in $56 \%$ of cases. Abnormal vital signs were noted in $21 \%$ of these, and etiologies were similar to those above, with the addition of cardiac arrest. Treatments used most were immobilization, oxygen, intravenous access, and several ALS medications. ALS was available in more than half the runs, but was used in only $14 \%$.

Conclusion: This multistate analysis confirms findings reported in smaller regional studies, with several exceptions. Excessive scene times were not noted. Few children had serious disorders as evidenced by abnormal vital signs and use of ALS. These findings have implications for EMS planners and educators.

\section{POSTER 008. \\ Accuracy of Priority Dispatch in an Urban EMS System}

James Dunford, $M D^{*}$

University of California-San Diego Medical Center, and the

San Diego Fire Department, San Diego, California USA

Purpose: To demonstrate a method of measuring the ability of priority dispatch protocols to predict the need for ALS intervention.

Methods: Retrospective analysis of computer-aided dispatch data from city of San Diego's EMS system. Dispatch priority (low vs high) and paramedic-assigned transport code acuity (10 $=$ acute, $20=$ ALS medication, $30=\mathrm{IV} / 02 /$ monitor, $40=$ BLS skills, 50 = transport only, CAN = cancel, DRY = nontransport) were analyzed.

Results: In 1994, of 69,539 medical aid requests, 846 were primarily triaged to BLS or police. The remaining 68,693 paramedic dispatches constituted the study population "low" priority assigned a no-code medic with a 15 -minute response $(11,736)$, while "high" priority assigned a code medic unit either alone $(8,348)$ or with an EMT-D first-responder $(48,609)$.

\begin{tabular}{|c|c|c|c|c|c|c|c|c|}
\hline de & CAN & RY & 10 & 20 & 30 & 40 & 50 & otals \\
\hline & 1,92 & & & & & & & \\
\hline & & & & & & & & \\
\hline & 244 & 5,518 & 4,486 & 5,062 & 13,832 & 9,379 & 8,172 & 8,69 \\
\hline
\end{tabular}

Ability to predict need for:

\begin{tabular}{|c|c|c|}
\hline & Code 10-20 & Code $10-30$ \\
\hline $\begin{array}{l}\text { SEN } \\
\text { SPEC } \\
\text { PPV } \\
\text { NPV }\end{array}$ & $\begin{array}{l}98.0 \\
19.5 \\
16.4 \\
98.3\end{array}$ & $\begin{array}{l}94.8 \\
23.2 \\
38.9 \\
89.6\end{array}$ \\
\hline
\end{tabular}

Conclusions: Current protocols are "safe" at the expense of significant over-triage. Only 194/11,736 (1.3\%) of low-priority dispatches required Code 10/20 transport. However, 45,313 $(68.7 \%)$ dispatches resulted in either Code $40,50, \mathrm{CAN}$ or DRY runs. This low prevalence of ALS-requiring conditions skews the protocols' positive predicative value. An acceptable "error rate" for EMS dispatch remains to be defined. This simple method may be useful when comparing EMS systems and in planning future managed-care needs. 